\title{
Lernen im Schlaf: Gleichstrom und Antidepressiva machen es möglich
}

\author{
Schlaf ist wichtig für die Gedächtniskonsolidierung. Diese lässt sich jedoch \\ gezielt manipulieren: mit Rosenduft, Gleichstrom und Noradrenalin-Wieder- \\ aufnahmehemmern.
}

$E_{\text {find }}^{\text {nat }}$ rinnerungen sind bekanntlich sehr flüchtig. Damit sie sich dauerhaft in das Gedächtnis eingraben, ist ein guter Schlaf unerlässlich. Bereits vor rund hundert Jahren haben Psychologen erkannt, dass Probanden Wörter besser lernen, wenn sie anschließend ein paar Stunden schlafen, statt wach zu bleiben. Inzwischen haben Forscher auch eine Ahnung, wie das geschieht. Dr. Susanne Diekelmann von der Universität Tübingen erinnerte etwa an Einzelzellableitungen bei Ratten: Laufen die Tiere einen Gang entlang, feuern an bestimmten Positionen im Kortex und Hippocampus spezifische Neurone, die für den Transfer von Inhalten in das Langzeitgedächtnis benötigt werden. Im Schlaf wiederholt sich dieses Aktivitätsmuster: Das Gehirn läuft den Gang praktisch noch einmal ab, ein Phänomen, das als "Replay“ bezeichnet wird.

\section{Rosenduft im Tiefschlaf}

Die Frage ist nun: Lässt sich das Replay verstärken? Das ist durchaus möglich,

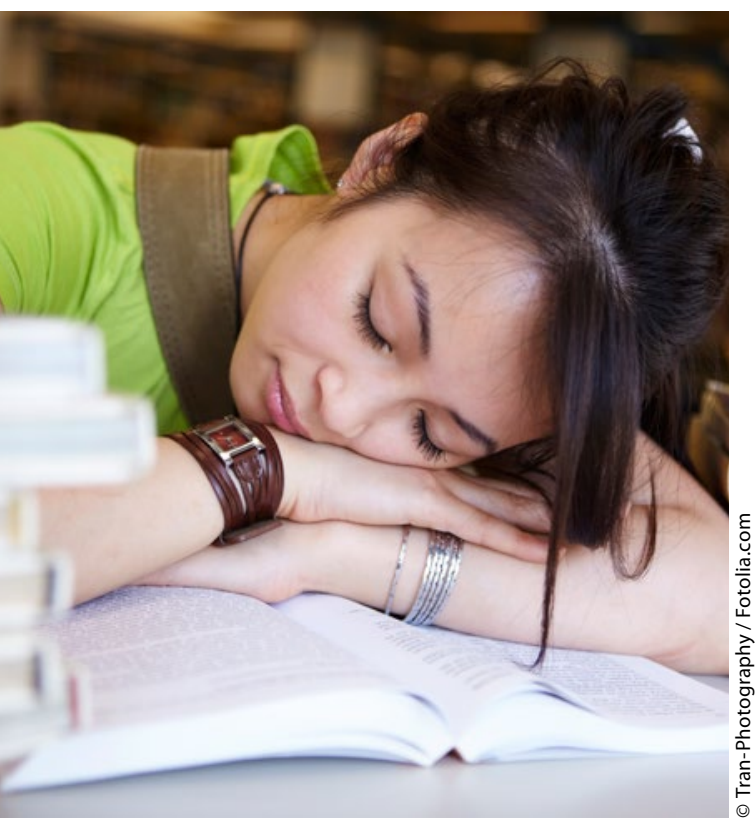

Sie festigt gerade das soeben Gelernte. wie Diekelmann anhand von eigenen Experimenten zeigen konnte. Ein Team vom Institut für Medizinische Psychologie und Verhaltensneurobiologie hatte Probanden gebeten, vor dem Schlaf Memory zu spielen. Sie sollten sich dabei die Positionen der Karten gut merken. „Wir haben den Probanden zugleich einen Rosenduft präsentiert, sodass der Geruch mit dem Memoryspiel verknüpft wurde“, erläuterte die Verhaltensforscherin. Im Schlaf haben die Tübinger Forscher ihre Versuchsteilnehmer erneute mit Rosenduft oder einem geruchslosen Placebo eingenebelt. Am nächsten Tag wurden dann einzelne Karten beim Memory aufgedeckt und die Probanden mussten sich an die korrekte Position der dazu passenden Karten erinnern. Wurde der Rosenduft im Tiefschlaf verströmt, konnten sich die Teilnehmer an signifikant mehr korrekte Kartenpositionen erinnern als unter Placeboduft. In anderen Schlafphasen zeigte der Duft hingegen keinen Effekt. Auch der Geruch allein ohne vorherige Verknüpfung mit dem Spiel führte zu keiner Gedächtnisverbesserung.

\section{Lernen im Rhythmus der Slow Oscillations}

Ein anderer Weg zum Neuroenhancement im Schlaf bietet die Manipulation von Schlafparametern, die an der Gedächtniskonsolidierung beteiligt sind. Im Wesentlichen, so Diekmann, seien das "Slow Oscillations“ aus dem Neokortex, also niederfrequente Schwingungen im Bereich von $0,8 \mathrm{~Hz}$, sowie "Sharp-Wave-Ripples“im Hippocampus - sie tragen vermutlich die Gedächtnisinhalte. Schließlich sorgen im Thalamus generierte Spindeln offenbar für ausreichend synaptische Plastizität. „Die zeitliche Kopplung dieser drei Parameter führt zum Transfer der Erinnerungen in das Langzeitgedächtnis“, erläuterte Diekmann.

Besonders einfach lassen sich die Slow Oscillations verstärken. Forscher konn- ten bereits vor neun Jahren zeigen, dass eine Gleichstromstimulation mit $0,8 \mathrm{~Hz}$ über dem präfrontalen Kortex während des Schlafs das Lernen von Wortlisten erleichtert. Im Vergleich zu einer Scheinstimulation konnten sich die Probanden am nächsten Tag an doppelt so viele Worte erinnern. „Das funktioniert nicht nur mit elektrischer, sondern auch mit auditorischer Stimulation: Wenn man bestimmte Töne im Rhythmus der Slow Oscillations präsentiert, führt auch das zu einer Verstärkung der Oszillationen und des Gedächtnisses.“

\section{Drehen am \\ Neurotransmittersystem}

Schließlich, so Diekmann, lasse sich die Gedächtniskonsolidierung auch über das Drehen am Neurotransmittersystem verbessern. Dazu eigenen sich unter anderem Antidepressiva wie Reboxetin, die die Verfügbarkeit von Noradrenalin erhöhen. Forscher der Universität Lübeck hatten Probanden gebeten, sich sechs verschiedene Düfte zu merken. Ein Teil von ihnen bekam über Nacht Reboxetin, die übrigen Placebo. Am nächsten Morgen wurden ihnen zu diesen sechs Düften sechs weitere präsentiert. Sie mussten nun entscheiden, welche sie schon am Vortag gerochen hatten und welche nicht. Das gelang in der Reboxetingruppe signifikant besser.

Insgesamt lässt sich also auf unterschiedlichen Wegen das Gedächtnis im Schlaf verbessern. Die Experimente lassen allerdings noch einige Fragen offen. So ist unklar, wie relevant die Effekte im Alltag sind und ob sie langfristig anhalten. Fraglich ist auch, ob Patienten mit kognitiven Defiziten davon profitieren. Zudem könnte es kognitive Nebenwirkungen geben. Vielleicht gehen die $\mathrm{Ma}$ nipulationen zulasten anderer Schlaffunktionen: Der Schlaf könnte weniger erholsam sein, wenn wir uns dabei zu viel mit dem Gedächtnis beschäftigen.

Thomas Müller

Symposium 8: Bedeutung des Schlafs für die kognitiven Fähigkeiten - die gesellschaftliche Perspektive. 23. Jahrestagung der DGSM, Mainz, 3. -5.12 .2015$ 